\title{
Closed Form Exact Solutions to the Higher Dimensional Fractional Schrodinger Equation via the Modified Simple Equation Method
}

\author{
M. Nurul Islam¹, M. Ali Akbar² \\ ${ }^{1}$ Department of Mathematics, Islamic University, Kushtia, Bangladesh \\ ${ }^{2}$ Department of Applied Mathematics, University of Rajshahi, Rajshahi, Bangladesh \\ Email: ali_math74@yahoo.com
}

How to cite this paper: Nurul Islam, M. and Ali Akbar, M. (2018) Closed Form Exact Solutions to the Higher Dimensional Fractional Schrodinger Equation via the Modified Simple Equation Method. Journal of Applied Mathematics and Physics, 6, 90-102.

https://doi.org/10.4236/jamp.2018.61009

Received: August 17, 2017

Accepted: January 8, 2018

Published: January 11, 2018

Copyright $\odot 2018$ by authors and Scientific Research Publishing Inc. This work is licensed under the Creative Commons Attribution International License (CC BY 4.0).

http://creativecommons.org/licenses/by/4.0/

c) (i) Open Access

\begin{abstract}
In this article, we investigate some exact wave solutions to the higher dimensional time-fractional Schrodinger equation, an important equation in quantum mechanics. The fractional Schrodinger equation further precisely describes the quantum state of a physical system changes in time. In order to determine the solutions a suitable transformation is considered to transmute the equations into a simpler ordinary differential equation (ODE) namely fractional complex transformation. We then use the modified simple equation (MSE) method to obtain new and further general exact wave solutions. The MSE method is more powerful and can be used in other works to establish completely new solutions for other kind of nonlinear fractional differential equations arising in mathematical physics. The affect of obtaining parameters for its definite values which are examined from the solutions of two dimensional and three dimensional time-fractional Schrodinger equations are discussed and therefore might be useful in different physical applications where the equations arise in this article.
\end{abstract}

\section{Keywords}

Modified Simple Equation (MSE) Method, Fractional Differential Equation, Nonlinear Evolution Equations, Higher Dimensional Schrodinger Equation, Traveling Wave Transformation

\section{Introduction}

The differential equations with fractional order have recently become a valuable tool to the modeling of numerous tangible events and it has gained importance and popularity to the researchers. The modeling of a tangible incident plays an 
important role on the history of the previous time which can also be successfully achieved by using fractional calculus. The use of fractional differentiation for the mathematical modeling of real-world physical problems has been widespread in recent years, for example, the modeling of earthquake, the fluid dynamic traffic model with fractional derivatives, and measurement of viscoelastic material properties. Applications of fractional differential equations in other fields, like quantum mechanics, electricity, plasma physics, chemical kinematics, optical fibers and related area are also felt. The fractional calculus has dominated in every field of sciences and engineering. In quantum mechanics the fractional Schrodinger equation [1] [2] is an equation that describes how the quantum state of a physical system changes in time. Thus, searching traveling wave solutions of fractional nonlinear evolution equations (NLEEs) plays a fundamental role. To know the internal mechanism of complex physical phenomena exact solutions of nonlinear fractional differential equations is very much important. As a result, recently some useful methods have been established and enhanced for obtaining exact solution to the fractional evolution equations such as, the extended direct algebraic function method [3] [4], the F-expansion method [5], the Adomian decomposition method [6], the homotopy perturbation method [7] [8] [9] [10], the tanh-function method [11], the Sine-Cosine method [12], the Jacobi elliptic method [13], the finite difference method [14], the variational iteration method [15] [16], the variational method [17], the Fourier transform technique [18], the modified decomposition method [19], the Laplace transform technique [20], the operational calculus method in [21], the exp-function method [22] [23], the $\left(G^{\prime} / G\right)$-expansion method [24] [25] [26], the modified simple equation method (MSE) [27]-[34], the $\exp (-\varphi(\eta))$-expansion method [35], the sub equation method [36], the multiple exp-function method [37] [38], the simplest equation method [39], the direct algebraic function method [40] [41] [42] [43], the extended auxiliary equation method [44] etc.

The aim of this article is to examine the further general and new exact solution of higher dimensional time-fractional Schrodinger equation by making use of the modified simple equation method [27]-[34] and discuss effect of the included parameters to the obtained solutions. We also discuss that the attained solutions might be useful and realistic to analyze the fractional quantum system for the time fractional two and three dimensional Schrodinger equation. We also have studied the behavior of emerging parameters which affect the obtained solutions and also describe how the quantum state of a physical system changes in time. In earlier literature, the time fractional Schrodinger equation is investigated through the first integral method [45], the F-expansion method [46], the Fourier transformation method [18], and the Laplace transformation method [47].

The MSE method is a recently developed efficient, potential and rising method to investigate wave solutions to the nonlinear fractional equations. Its finding results are straightforward, efficient, systematic, and no need to use the symbolic computation software to manipulate the algebraic equations. 
The rest of the article is processed as follows: In Section 2, we explain the Jumarie modified Riemann-Liouville derivative. In Section 3, we describe the outline of the MSE method. In Section 4, we investigate new and further general solutions to the time fractional equations mentioned above. In Section 5, we draw our conclusions.

\section{Modified Riemann-Liouville Derivative}

The Jumarie's modified Riemann-Liouville derivative of order $\alpha$ is defined as follows [48]:

$$
D_{x}^{\alpha} f(x)=\left\{\begin{array}{l}
\frac{1}{\Gamma(-\alpha)} \frac{\mathrm{d}}{\mathrm{d} x} \int_{0}^{x}(x-\xi)^{-\alpha-1}(f(\xi)-f(0)) \mathrm{d} \xi, \alpha<0, \\
\frac{1}{\Gamma(1-\alpha)} \frac{\mathrm{d}}{\mathrm{d} x} \int_{0}^{x}(x-\xi)^{-\alpha}(f(\xi)-f(0)) \mathrm{d} \xi, 0<\alpha<1, \\
{\left[f^{(\alpha-n)}(x)\right]^{(n)}, n \leq \alpha<n+1, n \geq 1 .}
\end{array}\right.
$$

Some properties for the proposed modified Riemann-Liouville derivative are listed [36] as follows:

$$
\begin{gathered}
D_{x}^{\alpha} x^{\gamma}=\frac{\Gamma(\gamma+1)}{\Gamma(\gamma+1-\alpha)} x^{\gamma-\alpha}, \gamma>0, \\
D_{x}^{\alpha}(f(x) g(x))=g(x) D_{x}^{\alpha} f(x)+f(x) D_{x}^{\alpha} g(x), \\
D_{x}^{\alpha} f[g(x)]=f_{g}[g(x)] D_{x}^{\alpha} g(x)=D_{g}^{\alpha} f[g(x)]\left(g^{\prime}(x)\right)^{\alpha},
\end{gathered}
$$

The above formulae play an important role in fractional calculus and also fractional differential equations.

\section{Outline of the Method}

Let us consider the nonlinear fractional evolution equation in the form:

$$
H\left(u, D_{t}^{\alpha} u, D_{x}^{\alpha} u, D_{t}^{2 \alpha} u, D_{x}^{2 \alpha} u, \cdots\right)=0,
$$

where $u=u(x, t)$ is wave function, $H$ is a polynomial in $u(x, t)$ and its partial derivatives, which consist of the highest order derivatives and nonlinear terms of the highest order, and the subscripts denote partial derivatives. To obtain the solution of (3.1) by using the MSE method [27]-[34], we have to execute the subsequent steps:

Step 1: We assume, $u(x, t)=\phi(\xi) \mathrm{e}^{i \eta}$ and the traveling wave variable,

$$
\xi=k\left(x-\frac{c t^{\alpha}}{\Gamma(1+\alpha)}\right) \text { and } \eta=m x-\frac{w t^{\alpha}}{\Gamma(1+\alpha)},
$$

permits us to transform the Equation (3.1) into the following ordinary differential equation (ODE):

$$
G\left(u, u^{\prime}, u^{\prime \prime}, \cdots\right)=0,
$$


where $G$ is a polynomial in $u(\xi)$ and its derivatives, wherein $u^{\prime}(\xi)=\frac{\mathrm{d} u}{\mathrm{~d} \xi}$.

Step 2: We assume that the solution of Equation (3.3) can be revealed in the form:

$$
u(\xi)=\sum_{i=0}^{N} a_{i}\left[\frac{s^{\prime}(\xi)}{s(\xi)}\right]^{i}
$$

where $a_{i},(i=0,1,2, \cdots, N)$ are unknown constants to be evaluated, such that $a_{N} \neq 0$, and $s(\xi)$ is an unidentified function to be estimated. In Jacobi elliptic function method, $\left(G^{\prime} / G\right)$-expansion method, F-expansion method, Riccati equation method, extended tanh-function method etc., the solutions are pre-defined or the solutions are presented in terms of some well-known differential equations, but in the MSE method, $s(\xi)$ is neither pre-defined nor a solution of any pre-defined differential equation. This is the individuality and uniqueness of the MSE method. Therefore, some useful and realistic solutions might be obtained by this method.

Step 3: The positive integer $N$ arises in Equation (3.4) can be determined by balancing the highest order nonlinear terms and the derivatives of highest order occur in Equation (3.4).

Step 4: Inserting (3.4) into (3.3) and simplifying for the function $s(\xi)$, we obtain a polynomial of $\left(\frac{1}{s(\xi)}\right)$. From the resulted polynomial, we equate all the coefficients of $(s(\xi))^{-i},(i=0,1,2, \cdots, N)$ to zero. This procedure yields a system of algebraic and differential equations which can be solved for determining $a_{i}(i=0,1,2, \cdots, N), s(\xi)$ and the other necessary parameters. This completes the determination of the solutions to the Equation (3.1).

\section{Applications of the Method}

In this section, we will examine the new and further general useful solutions to the time fractional $(2+1)$-dimensional and $(3+1)$-dimensional Schrodinger equations.

\subsection{The (2+1)-Dimensional Schrodinger Equation}

In this section, we investigate some applicable close form traveling wave solutions to the time fractional $(2+1)$-dimensional Schrodinger equation by making use the MSE method. Let us consider the time fractional $(2+1)$-dimensional Schrodinger equation of the form:

$$
i D_{t}^{\alpha} u=\frac{1}{2} u_{x x}\left(u_{x x}+u_{y y}\right)+p u+|u|^{2} u, \quad 0<\alpha \leq 1,
$$

where $\alpha$ and $p$ are emerging parameters. The Schrodinger equation is a mathematical equation that describes the variation over time of a physical structure on the fractional quantum system, as for instance wave particle duality is noteworthy. We can use this equation as a mathematical formula for the study 
of quantum mechanical system (The equation is a mathematical formula for the study of quantum mechanical systems). By means of the traveling transformation (3.2), the Equation (4.1.1) is converted into the following nonlinear ODE:

$$
(2 k m-c k) \phi^{\prime} i+\left(k^{2} \phi^{\prime \prime}+w \phi-m^{2} \phi-p \phi-\phi^{3}\right)=0,
$$

Equating real and imaginary part of Equation (4.1.2), we obtain

$$
(2 k m-c k) \phi^{\prime}=0,
$$

And

$$
k^{2} \phi^{\prime \prime}+w \phi-m^{2} \phi-p \phi-\phi^{3}=0 .
$$

As $\phi^{\prime} \neq 0$, from Equation (4.1.3), it can be easily obtained $c=2 m$.

Now, balancing the linear term of the highest order derivative term $\phi^{\prime \prime}$ and the nonlinear term of the highest order $\phi^{3}$ occurring in (4.1.4), yields $N=1$. Thus, the solution of Equation (4.1.4) is the form:

$$
\phi(\xi)=a_{0}+a_{1} \frac{s^{\prime}(\xi)}{s(\xi)},
$$

where $a_{0}$ and $a_{1}$ are constants to be determined, such that $a_{1} \neq 0$, and $s(\xi)$ is an unknown function to be evaluated. Now, it is simple to estimate the following:

$$
\begin{gathered}
\phi^{\prime}(\xi)=-\frac{a_{1}\left(s^{\prime}\right)^{2}}{s^{2}}+\frac{a_{1} s^{\prime \prime}}{s}, \\
\phi^{\prime \prime}(\xi)=\frac{2 a_{1}\left(s^{\prime}\right)^{3}}{s^{3}}-\frac{3 a_{1} s^{\prime} s^{\prime \prime}}{s^{2}}+\frac{a_{1} s^{\prime \prime \prime}}{s},
\end{gathered}
$$

Substituting the values of $\phi(\xi), \phi^{\prime \prime}(\xi)$ from (4.1.5) and (4.1.7) into (4.1.4) and then equating the coefficients of $s^{0}, s^{-1}, s^{-2}, s^{-3}, s^{-4}$ to zero, we respectively obtain

$$
\begin{gathered}
-m^{2} a_{0}-p a_{0}+w a_{0}+a_{0}^{3}=0 \\
-m^{2} a_{1} s^{\prime}(\xi)-p a_{1} s^{\prime}(\xi)+w a_{1} s^{\prime}(\xi)-3 a_{0}^{2} a_{1} s^{\prime}(\xi)+k^{2} a_{1} s^{\prime \prime \prime}(\xi)=0 \\
3 a_{0} a_{1}^{2} s^{\prime 2}(\xi)+3 k^{2} a_{1} s^{\prime}(\xi) s^{\prime \prime}(\xi)=0 \\
2 k^{2} a_{1} s^{\prime 3}(\xi)-a_{1}^{3} s^{\prime 3}(\xi)=0
\end{gathered}
$$

From (4.1.8) and (4.1.11), we attain

$$
a_{0}=0, a_{0}= \pm \sqrt{-m^{2}-p+w} \text { and } a_{1}= \pm \sqrt{2} k ; a_{1} \neq 0
$$

Case 1: When $a_{0}=0$, Equation (4.1.2) produces an absurd solution. Hence, the case is not accepted.

Case 2: When $a_{0}=\sqrt{-m^{2}-p+w}, a_{1}= \pm \sqrt{2} k$ and $c=2 m$, then from Equations. (4.1.9) and (4.1.10), we obtain

$$
s(\xi)=c_{1}-\frac{c_{2} k^{2}}{2 N} \mathrm{e}^{\mp 2 M \xi} \text {, where } M=\frac{\sqrt{-m^{2}-p+w}}{\sqrt{2} k}, N=m^{2}+p-w .
$$

Substituting the value of $a_{0}, a_{1}$ and $s(\xi)$ into the solution (4.1.5), it yields 


$$
\phi(\xi)=\frac{\sqrt{2} M k\left(c_{2} k^{2} \mathrm{e}^{\mp 2 M \xi}+2 c_{1} N\right)}{\left(-c_{2} k^{2} \mathrm{e}^{\mp 2 M \xi}+2 c_{1} N\right)}
$$

Converting the solution (4.1.12) from exponential to trigonometric function, we attain

$$
\phi(\xi)=\frac{\sqrt{2} M k\left(\left(c_{2} k^{2}+2 c_{1} N\right) \cosh (M \xi) \pm\left(-c_{2} k^{2}+2 c_{1} N\right) \sinh (M \xi)\right)}{\left(-c_{2} k^{2}+2 c_{1} N\right) \cosh (M \xi) \pm\left(c_{2} k^{2}+2 c_{1} N\right) \sinh (M \xi)}
$$

Since, $c_{1}$ and $c_{2}$ are arbitrary constant, one can choose their values arbitrarily. Therefore, if we choose, $c_{1}=k^{2}$ and $c_{2}= \pm 2 N$, from solution (4.1.13), we obtain

$$
\begin{aligned}
& \phi(\xi)= \pm \sqrt{2} M k \operatorname{coth}(M \xi) \\
& \phi(\xi)= \pm \sqrt{2} M k \tanh (M \xi)
\end{aligned}
$$

Again when $a_{0}=-\sqrt{-m^{2}-p+w}, a_{1}= \pm \sqrt{2} k$ and $c=2 m$, then from Equations (4.1.9) and (4.1.10), we obtain

$$
s(\xi)=c_{1}-\frac{c_{2} k^{2}}{2 N} \mathrm{e}^{ \pm 2 M \xi} \text {, where } M=\frac{\sqrt{-m^{2}-p+w}}{\sqrt{2} k}, N=m^{2}+p-w
$$

Inserting the values of $a_{0}, a_{1}$ and $s(\xi)$ into the solution (4.1.5), it yields

$$
\phi(\xi)=-\frac{\sqrt{2} M k\left(c_{2} k^{2} \mathrm{e}^{ \pm 2 M \xi}+2 c_{1} N\right)}{\left(-c_{2} k^{2} \mathrm{e}^{ \pm 2 M \xi}+2 N c_{1}\right)}
$$

Transforming the solution (4.1.16) from exponential to trigonometric function, it becomes

$$
\phi(\xi)=-\frac{\sqrt{2} M k\left(\left(c_{2} k^{2}+2 c_{1} N\right) \cosh (M \xi) \mp\left(-c_{2} k^{2}+2 c_{1} N\right) \sinh (M \xi)\right)}{\left(-c_{2} k^{2}+2 c_{1} N\right) \cosh (M \xi) \mp\left(c_{2} k^{2}+2 c_{1} N\right) \sinh (M \xi)}
$$

Here $c_{1}$ and $c_{2}$ are arbitrary constants, so one can select their values arbitrarily. Thus, if we select, $c_{1}=k^{2}$ and $c_{2}= \pm 2 N$, from the solution (4.1.17), we obtain

$$
\phi(\xi)= \pm \sqrt{2} M k \operatorname{coth}(M \xi)
$$

And

$$
\phi(\xi)= \pm \sqrt{2} M k \tanh (M \xi)
$$

Therefore, combining the solutions (4.1.14), (4.1.15), (4.1.18) and (4.1.19), we obtain the required solutions for this case as follows:

$$
\phi(\xi)= \pm \sqrt{2} M k \operatorname{coth}(M \xi)
$$

And

$$
\phi(\xi)= \pm \sqrt{2} M k \tanh (M \xi)
$$

Now, making use of the fractional wave variable (3.2) into solution (4.1.20), we obtain 


$$
\phi(\xi)= \pm \sqrt{2} M k \operatorname{coth}\left(M k\left(x-\frac{c t^{\alpha}}{\Gamma(1+\alpha)}\right)\right)
$$

And the solution (4.1.21), becomes

$$
\phi(\xi)= \pm \sqrt{2} M k \tanh \left(M k\left(x-\frac{c t^{\alpha}}{\Gamma(1+\alpha)}\right)\right)
$$

Substituting $M=\frac{\sqrt{-m^{2}-p+w}}{\sqrt{2} k}$, solution (4.1.22) yields

$$
\phi(\xi)= \pm \sqrt{-m^{2}-p+w} \operatorname{coth}\left(\frac{\sqrt{-m^{2}-p+w}}{\sqrt{2}}\left(x-\frac{c t^{\alpha}}{\Gamma(1+\alpha)}\right)\right)
$$

And the solution (4.1.23) yields

$$
\phi(\xi)= \pm \sqrt{-m^{2}-p+w} \tanh \left(\frac{\sqrt{-m^{2}-p+w}}{\sqrt{2}}\left(x-\frac{c t^{\alpha}}{\Gamma(1+\alpha)}\right)\right)
$$

The solutions attained in (4.1.24) and (4.1.25) are new and further general than the existing solutions. If we choose alternative values of $c_{1}$ and $c_{2}$, further closed form analytical solutions to the $(2+1)$-dimensional nonlinear time fractional Schrodinger equation can be extracted, but for simplicity and conciseness the remaining solutions have not been marked out.

\subsection{The (3+1)-Dimensional Schrodinger Equation}

In this section, we will use the MSE method to obtain new exact solution to the time-fractional two-dimensional Schrödinger equation. Consider the timefractional two-dimensional Schrödinger equation is of the form:

$$
i D_{t}^{\alpha} u=\frac{1}{2} u_{x x}\left(u_{x x}+u_{y y}+u_{z z}\right)+p u+|u|^{2} u, \quad 0<\alpha \leq 1
$$

where $\alpha$ and $p$ are emerging parameters. It arises as a description of the influence on the fractional quantum system. Using the traveling transformation (3.2), the Equation (4.2.1) becomes in the following nonlinear ODE:

$$
(6 k m-2 c k) \phi^{\prime} i+\left(3 k^{2} \phi^{\prime \prime}+2 w \phi-3 m^{2} \phi-2 p \phi-2 \phi^{3}\right)=0
$$

Separating real and imaginary part of Equation (4.2.2), we obtain

$$
(6 k m-2 c k) \phi^{\prime}=0,
$$

and

$$
\left(3 k^{2} \phi^{\prime \prime}+2 w \phi-3 m^{2} \phi-2 p \phi-2 \phi^{3}\right)=0 .
$$

Balancing the highest order derivative term $\phi^{\prime \prime}$ and the nonlinear term of the highest order $\phi^{3}$ occurring in (4.2.4) yields $N=1$. Thus, the solution of Equation (4.2.4) takes formal form:

$$
\phi(\xi)=a_{0}+a_{1} \frac{s^{\prime}(\xi)}{s(\xi)}
$$


where $a_{0}$ and $a_{1}$ are constants to be evaluated such that $a_{1} \neq 0$, and $s(\xi)$ is an unknown function to be determined. Now, it is simple to compute the followings:

$$
\begin{gathered}
\phi^{\prime}(\xi)=-\frac{a_{1}\left(s^{\prime}\right)^{2}}{s^{2}}+\frac{a_{1} s^{\prime \prime}}{s} \\
\phi^{\prime \prime}(\xi)=\frac{2 a_{1}\left(s^{\prime}\right)^{3}}{s^{3}}-\frac{3 a_{1} s^{\prime} s^{\prime \prime}}{s^{2}}+\frac{a_{1} s^{\prime \prime \prime}}{s}
\end{gathered}
$$

Inserting the values of $\phi(\xi), \phi^{\prime \prime}(\xi)$ from (4.2.5) and (4.2.7) into Equation (4.2.4) and then setting the coefficients of $s^{0}, s^{-1}, s^{-2}, s^{-3}, s^{-4}$ equal to zero, we respectively obtain

$$
\begin{gathered}
-3 m^{2} a_{0}-2 p a_{0}+2 w a_{0}-2 a_{0}^{3}=0 \\
-3 m^{2} a_{1} s^{\prime}(\xi)-2 p a_{1} s^{\prime}(\xi)+2 w a_{1} s^{\prime}(\xi)-6 a_{0}^{2} a_{1} s^{\prime}(\xi)+3 k^{2} a_{1} s^{\prime \prime \prime}(\xi)=0 \\
-6 a_{0} a_{1}^{2} s^{\prime 2}(\xi)-9 k^{2} a_{1} s^{\prime}(\xi) s^{\prime \prime}(\xi)=0 \\
6 k^{2} a_{1} s^{\prime 3}(\xi)-2 a_{1}^{3} s^{\prime 3}(\xi)=0
\end{gathered}
$$

As $\phi^{\prime} \neq 0$, from (4.2.3) it can be easily obtained $c=3 m$.

From Equations. (4.2.8) and Equation (4.2.11), we attain

$$
a_{0}=0, a_{0}= \pm \frac{\sqrt{-3 m^{2}-2 p+2 w}}{\sqrt{2}} \text { and } a_{1}= \pm \sqrt{3} k \text { since } a_{1} \neq 0 \text {. }
$$

Case 1: When $a_{0}=0$, Equation (4.2.2) provides an absurd solution. Hence, the case has not been accepted.

Case 2: When $a_{0}=\frac{\sqrt{-3 m^{2}-2 p+2 w}}{\sqrt{2}}, a_{1}= \pm \sqrt{3} k$ and $c=3 m$, from (4.2.9) and (4.2.10), we attain

$$
s(\xi)=c_{1}-\frac{3 k^{2} c_{2}}{N} \mathrm{e}^{ \pm 2 M \xi}, \text { where } M=\frac{\sqrt{-3 m^{2}-2 p+2 w}}{\sqrt{6} k}, \quad N=6 m^{2}+4 p-4 w
$$

Therefore, substituting the values of $a_{0}, a_{1}$ and $s(\xi)$ into the solution (4.2.5), we obtain

$$
\phi(\xi)=\frac{\sqrt{3} M k\left(3 c_{2} k^{2} \mathrm{e}^{\mp 2 M \xi}+N c_{1}\right)}{-3 c_{2} k^{2} \mathrm{e}^{\mp 2 M \xi}+N c_{1}}
$$

Converting the solution (4.2.12) from exponential to trigonometric function, we obtain

$$
\phi(\xi)=-\frac{\sqrt{3} M k\left(\left(3 c_{2} k^{2}+c_{1} N\right) \cosh (M \xi) \mp\left(3 c_{2} k^{2}-c_{1} N\right) \sinh (M \xi)\right)}{\left(3 c_{2} k^{2}-c_{1} N\right) \cosh (M \xi) \mp\left(3 c_{2} k^{2}+c_{1} N\right) \sinh (M \xi)}
$$

Here $c_{1}$ and $c_{2}$ are arbitrary constants. Since, $c_{1}$ and $c_{2}$ are arbitrary constants one might choose their values arbitrarily. Therefore, if we choose, $c_{1}=3 k^{2}$ and $c_{2}= \pm N$, from (4.2.13) we obtain

$$
\phi(\xi)= \pm \sqrt{3} M k \operatorname{coth}(M \xi)
$$

and 


$$
\phi(\xi)= \pm \sqrt{3} M k \tanh (M \xi)
$$

Again, when $a_{0}=-\frac{\sqrt{-3 m^{2}-2 p+2 w}}{\sqrt{2}}, a_{1}= \pm \sqrt{3} k$ and $c=3 m$, from (4.2.9) and (4.2.10) we obtain

$$
s(\xi)=c_{1}-\frac{3 k^{2} c_{2}}{N} \mathrm{e}^{ \pm 2 M \xi}, \text { where } M=\frac{\sqrt{-3 m^{2}-2 p+2 w}}{\sqrt{6} k}, N=6 m^{2}+4 p-4 w
$$

Thus, from (4.2.5) we obtain

$$
\phi(\xi)=\frac{\sqrt{3} M k\left(3 c_{2} k^{2}+c_{1} N \mathrm{e}^{2 M \xi}\right)}{\mp 3 c_{2} k^{2} \pm 0.5 c_{1} N \mathrm{e}^{2 M \xi}}
$$

Shifting the solution (4.2.16) from exponential to trigonometric function, we attain

$$
\phi(\xi)=\mp \frac{\sqrt{3} M k\left(\left(3 c_{2} k^{2}+c_{1} N\right) \cosh (M \xi)-\left(3 c_{2} k^{2}-c_{1} N\right) \sinh (M \xi)\right)}{\left(3 c_{2} k^{2}-c_{1} N\right) \cosh (M \xi)-\left(3 c_{2} k^{2}+c_{1} N\right) \sinh (M \xi)}
$$

where $c_{1}$ and $c_{2}$ are integral constants. Since $c_{1}$ and $c_{2}$ are arbitrary constants, so one might pick their values randomly. Now, if we pick, $c_{1}=3 k^{2}$ and $c_{2}= \pm N$, from (4.2.17) we obtain

$$
\phi(\xi)= \pm \sqrt{3} M k \operatorname{coth}(M \xi)
$$

and

$$
\phi(\xi)= \pm \sqrt{3} M k \tanh (M \xi)
$$

Therefore, comparing the solutions (4.2.14), (4.2.15), (4.2.18) and (4.2.19), we obtain the next solutions:

$$
\phi(\xi)= \pm \sqrt{3} M k \operatorname{coth}(M \xi)
$$

and

$$
\phi(\xi)= \pm \sqrt{3} M k \tanh (M \xi)
$$

Now, making use of the fractional wave variable (3.2) into solutions (4.2.20) and (4.2.21), we obtain

$$
\phi(\xi)= \pm \sqrt{3} M k \operatorname{coth}\left(M k\left(x-\frac{c t^{\alpha}}{\Gamma(1+\alpha)}\right)\right)
$$

and

$$
\phi(\xi)= \pm \sqrt{3} M k \tanh \left(M k\left(x-\frac{c t^{\alpha}}{\Gamma(1+\alpha)}\right)\right)
$$

Putting the value $M=\frac{\sqrt{-3 m^{2}-2 p+2 w}}{\sqrt{6} k}$, solutions (4.2.22) and (4.2.23) respectively become

$$
\phi(\xi)= \pm \frac{\sqrt{-3 m^{2}-2 p+2 w}}{\sqrt{2}} \operatorname{coth}\left(\frac{\sqrt{-3 m^{2}-2 p+2 w}}{\sqrt{6}}\left(x-\frac{c t^{\alpha}}{\Gamma(1+\alpha)}\right)\right)
$$


and

$$
\phi(\xi)= \pm \frac{\sqrt{-3 m^{2}-2 p+2 w}}{\sqrt{2}} \tanh \left(\frac{\sqrt{-3 m^{2}-2 p+2 w}}{\sqrt{6}}\left(x-\frac{c t^{\alpha}}{\Gamma(1+\alpha)}\right)\right)
$$

The solutions attained (4.2.24) and (4.2.25) are new and more general than the existing solutions. If we choose alternative values of $c_{1}$ and $c_{2}$, further closed form analytical solutions to the three dimensional nonlinear time-fractional Schrodinger equation can be extracted, but for simplicity and conciseness the residual solutions have not been marked out.

\section{Conclusion}

In this article, we have examined new and further general closed form solitons to time fractional two dimensional and three dimensional Schrodinger equations by means of the efficient technique known as modified simple equation (MSE) method. The solutions are attained in general form and definite values of the included parameters yield diverse known soliton solutions. The attained solutions might be useful to the influence on the fractional quantum system for the time fractional two dimensional and three dimensional Schrodinger equations. And we also have studied the behavior of emerging parameters which are affecting the physical system on the consider equations for the obtaining solutions. When the parameters take certain special values, the solitary waves are derived from the traveling waves. The established results show that the MSE method is more powerful, unified and can be used for many other fractional equations to get feasible solutions of the tangible incidents.

\section{References}

[1] Jiang, X., Qi, H. and Xu, M. (2011) Exact Solutions of Fractional Schrodinger-Like Equation with a Nonlocal Term. Journal of Mathematical Physics, 52, 042105. https://doi.org/10.1063/1.3576189

[2] Ablowitw, M.J. and Clarkson, P.A. (1991) Solutions, Nonlinear Evolution Equations and Inverse Scattering. Cambridge University Press, Cambridge. https://doi.org/10.1017/CBO9780511623998

[3] Seadawy, A.R. (2014) Stability Analysis for Zakharov-Kuznetsov Equation of Weakly Nonlinear Ion-Acoustic Wave in Plasma. Computers \& Mathematics with Applications, 67, 172-180. https://doi.org/10.1016/j.camwa.2013.11.001

[4] Seadaway, A.R. (2016) Ion Acoustic Solitary Wave Solutions of Two-Dimensional Nonlinear Kadomtsev-Petviashvili-Burgers Equation in Quantum Plasma. Mathematical Methods in the Applied Sciences, 40, 1598-1607. https://doi.org/10.1002/mma.4081

[5] Zhao, Y.M. (2013) F-Expansion Method and Its Application for Finding New Exact Solutions to the Kudryashov-Sinelshchikov Equation. Journal of Applied Mathematics, Article ID 895760, 7 p.

[6] El-Sayad, A.M.A., El-Kallad, I.L. and Ziada, E.A.A. (2010) Adomian Solution of Multi-Dimensional Nonlinear Equations of Arbitrary Orders. International Journal of Applied Mathematics and Mechanics, 6, 38-52. 
[7] Alomari, A.K. and Hashim, I. (2011) Analysis of Fully Developed Flow and Heat Transfer in a Vertical Channel with Prescribed Wall Heat Fluxes by the Homotopy Analysis Method. International Journal for Numerical Methods in Fluids, 67, 805-819. https://doi.org/10.1002/fld.2388

[8] Chen, Z. and Jiang, W. (2011) Piecewise Homotopy Perturbation Method for Solving Linear and Nonlinear Weakly Singular VIE of Second Order. Applied Mathematics and Computation, 217, 7790-7798.

https://doi.org/10.1016/j.amc.2011.02.086

[9] Mehrabinezhad, M. and Saberi-Nadjafi, J. (2010) Application of He's Homotopy Perturbation Method to Linear Programming Problem. International Journal of Computer Mathematics, 88, 341-347. https://doi.org/10.1080/00207160903443763

[10] Gupta, S., Kumar, D. and Singh, J. (2013) Application of He's Homotopy Perturbation Method for Solving Nonlinear Wave-Like Equations with Variable Coefficients. International Journal of Advances in Applied Mathematics and Mechanics, 1, 65-79.

[11] Wazwaz, A.M. (2004) The Tanh-Function Method for Travelling Wave Solutions of Nonlinear Equations. Applied Mathematics and Computation, 154, 713-723. https://doi.org/10.1016/S0096-3003(03)00745-8

[12] Jawad, A.J.M. (2012) The Sine-Cosine Function Method for the Exact Solutions of Nonlinear Partial Differential Equations. International Journal of Recent Research and Applied Studies, 13, 186-191.

[13] Zheng, B. and Feng, Q. (2014) The Jacobi Elliptic Equation Method for Solving Fractional Partial Differential Equations. Abstract and Applied Analysis, 2014, Article ID 249071, 9 p.

[14] Dehghan, M. (2000) A Finite Difference Method for a Non-Local Boundary Value Problem for Two Dimensional Heat Equations. Applied Mathematics and Computation, 112, 133-142. https://doi.org/10.1016/S0096-3003(99)00055-7

[15] Wazwaz, A.M. (2007) The Variational Iteration Method for Analytic Treatment for Linear and Nonlinear ODEs. Applied Mathematics and Computation, 212, 120-134. https://doi.org/10.1016/j.amc.2009.02.003

[16] Wazwaz, A.M. (2007) The Variational Iteration Method: A Powerful Scheme for Handling Linear and Nonlinear Diffusion Equations. Computers \& Mathematics with Applications, 54, 933-939. https://doi.org/10.1016/j.camwa.2006.12.039

[17] Helal, M.A. and Seadawy, A.R. (2009) Variational Method for the Derivative Nonlinear Schrodinger Equation with Computational Applications. Physica Scripta, 80, Article ID: 035004.

[18] Glushkov, E., Glushkov, N. and Chen, C.S. (2007) Semi Analytical Solution to Heat Transfer Problems using Fourier Transform Technique, Radial Basis Functions, and the Method of Fundamental Solutions. Numerical Heat Transfer, Part B: Fundamentals, 52, 1-28. https://doi.org/10.1080/10407790701443859

[19] Wazwaz, A.M. (2006) The Modified Decomposition Method and Pade Approximants for a Boundary Layer Equation in Unbounded Domain. Applied Mathematics and Computation, 177, 737-744. https://doi.org/10.1016/j.amc.2005.09.102

[20] Abassy, T.A., El-Tawil, M.A. and El-Zoheiry, H. (2007) Exact Solution of Some Nonlinear Partial Differential Equations using the Variational Iteration Method Linked with Laplace Transforms and the Pade Technique. Computers \& Mathematics with Applications, 54, 940-954. https://doi.org/10.1016/j.camwa.2006.12.067

[21] Hilfer, R., Luchko, Y. and Tomovski, Z. (2009) Operational Method for the Solution of Fractional Differential Equations with Generalized Riemann-Liouville Fractional 
Derivatives. Fractional Calculus and Applied Analysis, 12, 299-318.

[22] Dai, C.Q. and Zhang, J.F. (2009) Application of He's Exp-Function Method to the Stochastic mKdV Equation. International Journal of Nonlinear Sciences and $\mathrm{Nu}$ merical Simulation, 10, 675-680. https://doi.org/10.1515/IJNSNS.2009.10.5.675

[23] Zhang, W.M. and Tian, L.X. (2009) Generalized Solitary Solution and Periodic Solution of the Combined KdV-mKdV Equation with Variable Coefficients using the Exp-Function Method. International Journal of Nonlinear Sciences and Numerical Simulation, 10, 711-715. https://doi.org/10.1515/IJNSNS.2009.10.6.711

[24] Akbar, M.A., Ali, N.H.M. and Zayed, E.M.E. (2012) A Generalized and Improved $\left(G^{\prime} / G\right)$-Expansion Method for Nonlinear Evolution Equations. Mathematical Problems in Engineering, 2012, Article ID: 459879. https://doi.org/10.1155/2012/459879

[25] Zhang, S., Tong, J. and Wang, W. (2008) A Generalized $\left(G^{\prime} / G\right)$-Expansion Method for the mKdV Equation with Variable Coefficients. Physics Letters A, 372, 2254-2257. https://doi.org/10.1016/j.physleta.2007.11.026

[26] Alam, M.N. and Akbar, M.A. (2014) Application of the New Approach of Generalized $\left(G^{\prime} / G\right)$-Expansion Method to Find Exact Solutions of Nonlinear PDEs in Mathematical Physics. BIBECHANA, 10, 58-70.

[27] Bekir, A., Kaplan, M. and Guner, O. (2015) A Novel Modified Simple Equation Method and Its Application to Some Nonlinear Evolution Equation System. AIP Conference Proceedings, 1611, 30-36. https://doi.org/10.1063/1.4893799

[28] Zayed, E.M.E. (2013) The Enhanced Modified Simple Equation Method for Solving Nonlinear Evolution Equations with Variable Coefficients. AIP Conference Proceeding, 1558, 1999-2004.

[29] Akther, J. and Akbar, M.A. (2016) Solitary Wave Solution to Two Nonlinear Evolution Equations via the Modified Simple Equation Method. New Trends in Mathematical Sciences, 4, 12-26. https://doi.org/10.20852/ntmsci.2016422033

[30] Ayati, Z., Moradi, M. and Mirzazadeh, M. (2015) Application of Modified Simple Equation Method to Burgers, Huxley and Burgers-Huxeley Equations. Iranian Journal of Numerical Analysis and Optimization, 5, 59-73.

[31] Zayed, E.M.E. and Ai-Nowehy, A.G. (2016) The Modified Simple Equation Method, the Exp-Function Method, and the Method of Solution Ansatz for Solving the Long-Short Wave Resonance Equations. Zeitschrift für Naturforschung A, 71, 10.

[32] Khan, M.A., Akbar, M.A. and Belgacem, F.B.M. (2016) Solitary Wave Solutions for the Boussinesq and Fisher Equations by the Modified Simple Equation Method. Mathematics Letters, 2, 1-18.

[33] Zayed, E.M.E., Amer, Y.A. and Al-Nowehy, A.G. (2016) The Modified Simple Equation Method and the Multiple Ex-Function Method for Solving Nonlinear Fractional Sharma-Tasso-Olver Equation. Acta Mathematicae Applicatae Sinica, 32, 793-812. https://doi.org/10.1007/s10255-016-0590-9

[34] Khan, K. and Akbar, M.A. (2013) Exact and Solitary Wave Solutions for the Tzitzeica-Dodd Bullough and the Modified KdV-Zakharov-Kuznetsov Equations using the Modified Simple Equation Method. Ain Shams Engineering Journal, 4, 903-909. https://doi.org/10.1016/j.asej.2013.01.010

[35] Ali, A., Iqbal, M.A. and Mohyud-Din, S.T. (2016) Traveling Wave Solutions of Generalized Zakharov-Kuznetsov-Benjamin-Bona-Mahony and Simplified Modified Form of Camassa-Holm Equation Method by using $\exp (-\phi(\eta))$ Method. Egyp- 
tian Journal of Basic and Applied Sciences, 3, 134-140.

https://doi.org/10.1016/j.ejbas.2016.01.001

[36] Yasar, E., Yildirim, Y. and Khalique, C.M. (2016) Lie Symmetry Analysis, Conservation Laws and Exact Solutions of the Seventh-Order Time Fractional Sawada-Kotera-Ito Equation. Results in Physics, 6, 322-328.

https://doi.org/10.1016/j.rinp.2016.06.003

[37] Yildirim, Y., Yasar, E. and Adem, A.R. (2017) A Multiple Exp-Function Method for the Three Model Equations of Shallow Water Waves. Nonlinear Dynamics, 89, 2291-2297. https://doi.org/10.1007/s11071-017-3588-9

[38] Yildirim, Y. and Yasar, E. (2017) Multiple Exp-Function Method for Soliton Solutions of Nomlinear Evolution Equations. Chinese Physics B, 26, Article ID: 070201. https://doi.org/10.1088/1674-1056/26/7/070201

[39] Giresunlu, I.B., Ozkan, Y.S. and Yasar, E. (2017) On the Exact Solutions, Lie Symmetry Analysis, and Conservation Laws of Schamel-Korteweg-de Vries Equation. John Wiley Sons Ltd., Hoboken.

[40] Seadawy, A.R. (2015) Fractional Solitary Wave Solutions of the Nonlinear Higher-Order Extended KdV Equation in a Stratified Shear Flow: Part I. Computers \& Mathematics with Applications, 70, 345-352. https://doi.org/10.1016/j.camwa.2015.04.015

[41] Seadawy, A.R. (2015) Three-Dimensional Nonlinear Modified Zakharov-Kuznetsov Equation of Ion-Acoustic Waves in a Magnetized Plasma. Computers \& Mathematics with Applications, 71, 201-212.

[42] Seadawy, A.R. and El-Rashidy, K. (2013) Traveling Wave Solutions for Some Coupled Nonlinear Evolution Equations. Mathematical and Computer Modelling, 57, 1371-1379. https://doi.org/10.1016/j.mcm.2012.11.026

[43] Seadawy, A.R. (2016) Stability Analysis Solutions of Nonlinear Three-Dimensional Modified Kortweg-de Vries-Zakharov-Kuznetsov Equation in Magnetized Electron-Positron Plasma. Physica A, 455, 44-51. https://doi.org/10.1016/j.physa.2016.02.061

[44] Seadawy, A.R. (2017) Traveling Wave Solutions of a Weakly Nonlinear Two-Dimensional and High-Order Kadompsev-Petviashvili Dynamical Equation for Dispersive Shallow Water. The European Physical Journal Plus, 132, 29. https://doi.org/10.1140/epjp/i2017-11313-4

[45] Moosaei, H., Mirzazadeh, M. and Yildirim, A. (2011) Exact Solutions to the Perturbed Nonlinear Schrodinger's Equation with Kerr Law Nonlinearity by using the First Integral Method. Nonlinear Analysis: Modelling and Control, 16, 332-339.

[46] Filiz, A., Ekici, M. and Sonmezoglu, A. (2014) F-Expansion Method and New Exact Solutions of the Schrodinger-KdV Equation. The Scientific World Journal, 2014, Article ID: 534063. https://doi.org/10.1155/2014/534063

[47] El-Tawil, A. and El-Zoheiry, H. (2007) Exact Solutions of Some Nonlinear Partial Differential Equations using the Variational Iteration Method Linked with Laplace Transforms and the Pade Technique. Computers \& Mathematics with Applications, 54, 940-954. https://doi.org/10.1016/j.camwa.2006.12.067

[48] Jumarie, G. (2006) Modified Riemann-Liouville Derivative and Fractional Taylor Series of Non-Differentiable Functions Further Results. Computers \& Mathematics with Applications, 51, 1367-1376. https://doi.org/10.1016/j.camwa.2006.02.001 